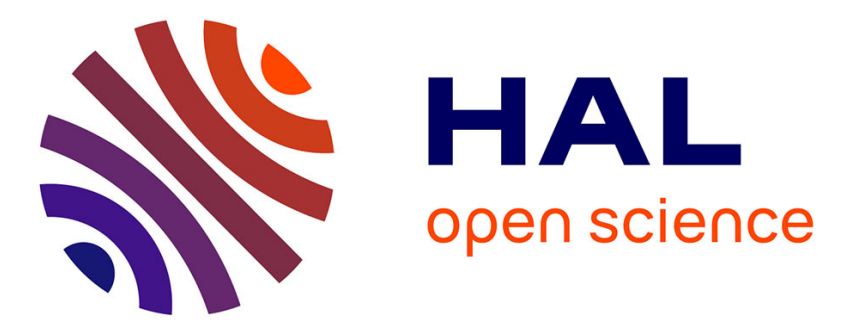

\title{
Random-energy model: limit of a family of disordered models
}

\author{
Bernard Derrida
}

\section{To cite this version:}

Bernard Derrida. Random-energy model: limit of a family of disordered models. Physical Review Letters, 1980, 45 (2), pp.79-82. 10.1103/PhysRevLett.45.79 . hal-03285940

\author{
HAL Id: hal-03285940 \\ https://hal.science/hal-03285940
}

Submitted on 21 Jul 2021

HAL is a multi-disciplinary open access archive for the deposit and dissemination of scientific research documents, whether they are published or not. The documents may come from teaching and research institutions in France or abroad, or from public or private research centers.
L'archive ouverte pluridisciplinaire HAL, est destinée au dépôt et à la diffusion de documents scientifiques de niveau recherche, publiés ou non, émanant des établissements d'enseignement et de recherche français ou étrangers, des laboratoires publics ou privés. 
1977), p. 351 .

${ }^{9}$ W. A. Edelstein, J. Hough, J. R. Pugh, and W. Martin, J. Phys. E 11, $710(1978)$.

${ }^{10}$ W. G. Unruh, in Proceedings of an Astrophysics
Summer School on Gravitational Radiation and Collapsed Objects, Nedlands, Western Australia, 1979, edited by C. Edwards (Springer, Berlin, 1980).

${ }^{11}$ R. Loudon, Phys. Bull. 27, 21 (1976).

\title{
Random-Energy Model: Limit of a Family of Disordered Models
}

\author{
B. Derrida \\ Service de Physique Théorique, Centre d'Etudes Nucléaires de Saclay, 91190 Gif-sur-Yvette, France
}

(Received 9 April 1980)

\begin{abstract}
In this Letter, a simple model of disordered systems - the random-energy model-is introduced and solved. This model is the limit of a family of disordered models, when the correlations between the energy levels become negligible. The properties are qualitatively the same as those of the Sherrington-Kirkpatrick model. Moreover, this random-energy model looks like a simple approximation to any spin-glass model.
\end{abstract}

PACS numbers: $05.50 .+\mathrm{q}, 64.60 . \mathrm{Cn}, 75.50 . \mathrm{Kj}$

Recently, a lot of effort has been devoted to solving the Sherrington-Kirkpatrick model (the S.K. model). ${ }^{1}$ This model was introduced to enable one to understand the properties of the Edwards-Anderson spin-glass model $^{2}$ in the case where the range of the interactions is infinite and where, therefore, a mean-field theory ${ }^{3}$ for spin glass models would be exact. Though it is now accepted that the failure of the solution initially proposed is due to a breaking of symmetry in the replica space, ${ }^{4}$ and in spite of the effort displayed ${ }^{5,6}$ to find new solutions of the S.K. model, no simple analytic solution has yet been proposed.

In this Letter, I introduce and solve a new model of disordered systems, the random-energy model. This model describes a system whose energy levels are independent random variables. Many of its properties are very similar to those of the S.K. model: the same qualitative phase diagram, the same free energy in the high temperature phase, the same kind of corrections to the thermodynamic limit. It gives a simplified picture of a transition in a disordered system: The specific heat vanishes in the whole low-temperature phase and the system becomes completely frozen below its critical temperature.

This random-energy model is the limit of a family of models with random interactions which generalize the S.K. model. To describe these models, we consider a system of $N$ interacting Ising spins with infinite-ranged random $p$-spin interactions. For such a model, the Hamiltonian $\mathcal{H}_{p}$ can be written

$$
\mathcal{H}_{p}(\{\sigma\})=-\sum_{\left(i_{1}, i_{2}, \ldots, i_{p}\right)} A_{i_{1} i_{2} \ldots i_{p}} \sigma_{i_{1}} \ldots \sigma_{i_{p}},
$$

where in $\mathfrak{H}_{p}$, there is a random interaction $A_{i_{1} \ldots i_{p}}$ for any group of $p$ spins in the system. In order to ensure an extensive thermodynamic limit, one has to scale properly the probability distribution of the interactions $A_{i_{1} \ldots i_{p}}$ with $N$. I choose here Gaussian distributions,

$$
\rho\left(A_{i_{1} \ldots i_{p}}\right)=\left(N^{p-1} / \pi J^{2} p !\right)^{1 / 2} \exp \left[-\left(A_{i_{1} \ldots i_{p}}\right)^{2} N^{p-1} / J^{2} p !\right],
$$

where $p=1$ corresponds to a system of free spins in a random magnetic field, and $p=2$ is the S.K. model.

To establish the relation between all these models, I introduce the one-level probability distribution $P(E)$ as the probability that a given configuration of the spins $\left\{\sigma_{i}{ }^{(1)}\right\}$, say configuration (1), has a given energy:

$$
P(E)=\left\langle\delta\left(E-\mathcal{H}\left(\left\{\sigma^{(1)}\right\}\right)\right)\right\rangle,
$$

where in (3) the average is taken over all the possible choices of the interactions $A_{i_{1} \ldots i_{p}}$. In the same way, one can define the two-level probability distribution $P\left(E_{1}, E_{2}\right)$ as the probability that two given 
configurations $\left\{\sigma_{i}^{(1)}\right\}$ and $\left\{\sigma_{i}^{(2)}\right\}$ have, respectively, energies $E_{1}$ and $E_{2}$ :

$$
P\left(E_{1}, E_{2}\right)=\left\langle\delta\left(E_{1}-\mathcal{H C}\left(\left\{\sigma^{(1)}\right\}\right)\right) \delta\left(E_{2}-\mathcal{H}\left(\left\{\sigma^{(2)}\right\}\right)\right)\right\rangle .
$$

Using Eqs. (1) and (2), one gets in the large- $N$ limit

$$
\begin{aligned}
& P(E) \sim\left(N \pi J^{2}\right)^{-1 / 2} \exp \left(-E^{2} / N J^{2}\right), \\
& P\left(E_{1}, E_{2}\right) \sim C \exp \left[-\frac{\left(E_{1}+E_{2}\right)^{2}}{2 N\left[1+(2 x-1)^{p}\right] J^{2}}-\frac{\left(E_{1}-E_{2}\right)^{2}}{2 N\left[1-(2 x-1)^{p}\right] J^{2}}\right] .
\end{aligned}
$$

It should be noticed that $P\left(E_{1}, E_{2}\right)$ depends not only on the energies but also on the number $x$ where $N x$ is the number of identical spins in the two configurations; $p$ refers to the model defined by Eqs. (1) and (2) that we consider and $C$ is a normalization factor. The reason why $P(E)$ does not depend explicitly on the spin configuration is that the probability distribution $\rho(A)$ of the $p$-spin interaction is symmetric (or gauge invariant ${ }^{7}$ ). Because of the presence of infinite-ranged interactions, $P\left(E_{1}, E_{2}\right)$ depends only on the number $N x$ of identical spins in the two configurations. The expression for $P\left(E_{1}, E_{2}\right)$ tells us how the energies of different configurations of spins are correlated. When $x \rightarrow 1$, that is to say when the two configurations are almost identical, the two energies $E_{1}$ and $E_{2}$ are very closely correlated and $P\left(E_{1}, E_{2}\right)$ $\sim P\left(E_{1}\right) \delta\left(E_{1}-E_{2}\right)$. In contrast, when $x \sim \frac{1}{2}$, Eq. (5) gives $P\left(E_{1}, E_{2}\right) \sim P\left(E_{1}\right) P\left(E_{2}\right)$ and the two energies are completely uncorrelated. The fact to be noticed is that when $p$ increases, the energies of two different configurations become independent random variables because $(2 x-1)^{p} \rightarrow 0$ when $p \rightarrow \infty$ if $|x|<1$, and then $P\left(E_{1}, E_{2}\right) \sim P\left(E_{1}\right) P\left(E_{2}\right)$ for any pair of configurations. This leads me to introduce the random-energy model as the model we obtain when $p$ is large and when, therefore, the energy levels are independent random variables.

The random-energy model is defined as a system which has the following three properties:

(i) The system has $2^{N}$ energy levels $E_{i}$. (ii) The energy levels $E_{i}$ are random variables distributed according to the probability law

$$
P(E)=\left(N \pi J^{2}\right)^{-1 / 2} \exp \left(-E^{2} / N J^{2}\right) .
$$

(iii) The $E_{i}$ are independent random variables.

The first two properties are actual features of many spin-glass models, especially of all the models defined by the Hamiltonian (1). The role of the third property is to simplify the model to allow us to solve it exactly. The partition function $Z$ for a given sample of the $2^{N}$ energies $E_{i}$ is

$$
Z\left(\left\{E_{i}\right\}\right)=\sum_{i=1}^{2^{N}} \exp \left(-\frac{E_{i}}{T}\right)
$$

and as usual, for quenched disordered systems, one wants to calculate the average free energy:

$$
F=-T\langle\ln Z\rangle=-T \int \Pi_{i}\left[P\left(E_{i}\right) d E_{i}\right] \ln Z\left(\left\{E_{i}\right\}\right) .
$$

The simplest way to solve this model is to use the microcanonical language. For one sample of $2^{N}$ energy levels, we call $n(E)$ the number of energy levels belonging to the interval $(E, E+d E)$. This number fluctuates from one sample to another. However, the average $\langle n(E)\rangle$ over all the choices of the energies $E_{i}$ follows directly from (ii):

$$
\begin{aligned}
\langle n(E)\rangle & =2^{N} P(E) \\
& \sim \exp \left\{N\left[\ln 2-(E / N J)^{2}\right]\right\} .
\end{aligned}
$$

It is then clear that there exists a critical energy $E_{0}$ defined by

$$
E_{0} / N=J(\ln 2)^{1 / 2} \text {. }
$$

If $|E|<E_{0}$, the average number of levels is much larger than 1. Because of the statistical independence of the energy levels, this means that for almost all the samples, the fluctuations of $n(E)$ are of order $\langle n(E)\rangle^{1 / 2}$. Therefore

$$
n(E) \sim\langle n(E)\rangle \text {. }
$$

If $|E|>E_{0},\langle n(E)\rangle$ is much smaller than 1. So for almost all the samples $n(E)=0$ and with a very small probability $n(E) \geqslant 1$. This means that with probability 1 , there is not even one of the $2^{N}$ energies $E_{i}$ larger than $E_{0}$ or smaller than $-E_{0}$. So with probability 1 , when $N$ is larger, the entropy $S(E)$ is given by

$$
\begin{aligned}
S(E) \simeq \ln & \langle n(E)\rangle \\
& =N\left[\ln 2-(E / N J)^{2}\right] \text { if }|E|<E_{0},
\end{aligned}
$$

and there is no energy level larger than $E_{0}$ in absolute value.

To calculate the free energy, one has to find the point on the curve $S(E)$ where the slope $d S / d E$ $=1 / T$ (Fig. 1 ).

It is then clear that this random-energy model 


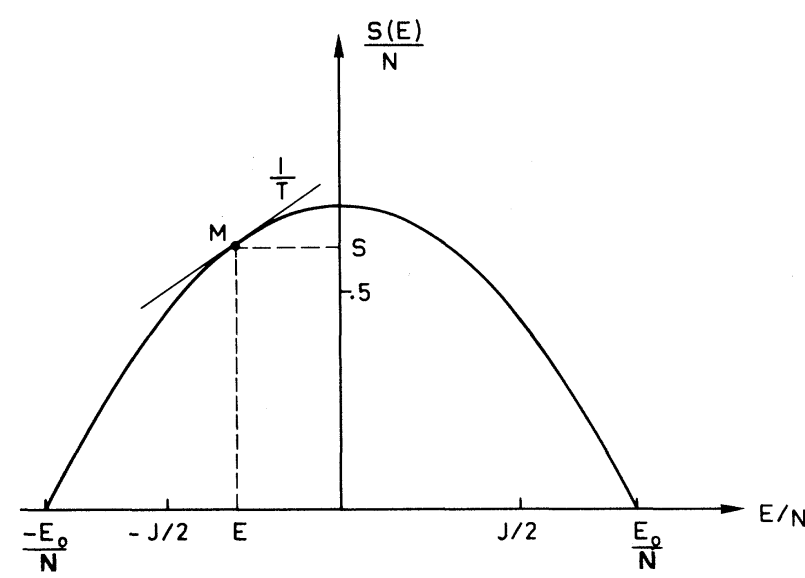

FIG. 1. The entropy $S(E)$ as a function of energy for the random-energy model.

has a critical temperature $T_{c}=J / 2(\ln 2)^{1 / 2}$ which is related to the slope of $S(E)$ at the point $E=-E_{0}$. If $T>T_{c}$, the free energy is

$$
N^{-1} F=-T \ln 2-J^{2} / 4 T
$$

and the point $M$ of Fig. 1 moves on the curve $S(E)$ when $T$ changes. If $T<T_{c}$, the point $M$ sticks to $-E_{0}$ and then

$$
N^{-1} F=-E_{0} / N=-J(\ln 2)^{1 / 2} \text {. }
$$

So below $T_{c}$, the system is frozen in its ground state and the specific heat vanishes in the whole low-temperature phase. This result can be compared with the predictions of mean-field theory for ferromagnetic systems where the specific heat vanishes in the whole high temperature phase. ${ }^{8}$

More sophisticated calculations ${ }^{9}$ can be done to obtain the corrections to the thermodynamic limit for this random-energy model. For the groundstate energy of this random-energy model, one finds $N^{-1} \ln N$ corrections which are comparable to the $1 / N$ (Ref. 1) or $1 / \sqrt{N}$ (Ref. 10) corrections conjectured for the S.K. model. Moreover, as for the S.K. model, the transition temperature appears as a divergence of the subdominant terms.

An interesting question is the behavior of this random-energy model in a uniform magnetic field $H$. To define the model, one has to come back to the Hamiltonians $\mathfrak{H}_{p}$. In presence of a field $H$, the Hamiltonians become

$$
\mathfrak{H}_{p}{ }^{\prime}=\mathfrak{H}_{p}-H \sum_{i} \sigma_{i} .
$$

One can calculate again the probability distributions $P(E)$ and $P\left(E_{1}, E_{2}\right)$. As before, $P\left(E_{1}, E_{2}\right)$ is factorized and the energy levels become independent when $p \rightarrow \infty$. The difference is that now, $P(E)$ depends on the configuration, more precisely on its magnetization $M$ :

$$
P(E) \sim C \exp \left[-(E+M H)^{2} / N J^{2}\right] .
$$

So the random energy model is a system of $2^{N}$ independent random energy levels among which $\left(\begin{array}{c}N \\ (N+M) / 2\end{array}\right)$ have a magnetization $M$ and are distributed according to (12). One can then use the same arguments as before to calculate the average free energy: The entropy is $\ln \langle n(E)\rangle$ when $\langle n(E)\rangle$ is large and there is no energy level when $\langle n(E)\rangle$ is small. This argument remains true because the energies are independent variables. For any value of the field, there is a phase transition at a critical temperature $T_{c}(H)$ and the low-temperature phase is always frozen with a magnetization independent of temperature. ${ }^{9}$ From the free energy, one finds the susceptibility $\chi$ for $H=0$ :

$$
\begin{aligned}
& \chi=1 / T \text { if } T>T_{c}, \\
& \chi=1 / T_{c} \text { if } T<T_{c} .
\end{aligned}
$$

A similar constant susceptibility at low temperature was also predicted by Parisi ${ }^{11}$ for the S.K. model.

To continue the comparison with the S.K. model, one can study the case where in addition to the random interactions, there is a ferromagnetic pair interaction. The new Hamiltonians are

$$
\mathcal{H}_{p} \prime=\mathcal{H}_{p}-\frac{J_{0}}{N} \sum_{\langle i j\rangle} \sigma_{i} \sigma_{j} \cdot
$$

Here again, we can use the same arguments to study the large- $p$ limit of these problems. The results ${ }^{9}$ are summarized in the phase diagram (Fig. 2). If $J_{0}<J / 2(\ln 2)^{1 / 2}$, there is only one phase transition. In the low-temperature phase (I), the system is completely frozen (nothing depends on temperature) and the magnetization is zero. If $J_{0}>J / 2(\ln 2)^{1 / 2}$, the low-temperature phase (II) is also completely frozen but with a finite magnetization. When $T$ increases, there is at first a transition to a ferromagnetic phase and then another transition to the paramagnetic phase. Again the same kind of phase diagram was found for the S.K. model. ${ }^{1}$

In this Letter, I have solved this random-energy model for which the energy levels are taken as independent random variables. This model is a simple approximation to more realistic spinglass models as the correlations between the energy levels are ignored. It would be very interest- 


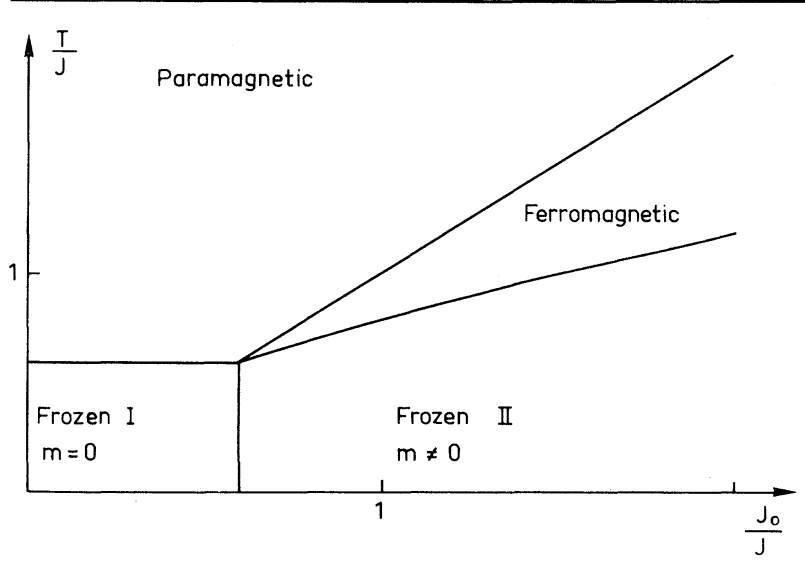

FIG. 2. The phase diagram of the random-energy model in presence of ferromagnetic pair interactions.

ing to improve this approximation by trying to include these correlations. One possibility may be to look at the models defined by Eq. (1) when $p$ is large. In spite of its simplicity, this model leads to the same qualitative predictions as the S.K. model. So it seems to contain some physics and it is a good example to help one understand the origin of the difficulties one usually encounters in spin glass problems.

I wish to thank the Groupe de Physique des So- lidas of the Ecole Normale Superrieure and the Division de Physique Theorique, especially P. Moussa, Y. Pomeau, G. Toulouse, and J. Vannimenus, for many stimulating discussions.

${ }^{1}$ D. Sherrington and S. Kirkpatrick, Phys. Rev. Lett. 35, 1972 (1975), and Phys. Rev. B 17, 4384 (1978).

${ }^{2}$ S. F. Edwards and P. W. Anderson, J. Phys. F $\underline{5}$, 965 (1975).

${ }^{3}$ D. J. Thouless, P. W. Anderson, and R. G. Palmer, Philos. Mag. 35, 593 (1977).

${ }^{4}$ J. R. L. de Almeida and D. J. Thouless, J. Phys. A 11, 983 (1978).

${ }^{5}$ A. Blandin, M. Gabay, and T. Garel, J. Phys. C $\underline{13}$, 403 (1980).

${ }^{6}$ G. Parisi, J. Phys. A 13 , 1101 (1980).

${ }^{7}$ G. Toulouse, Commun. Phys. 2, 115 (1977); J. Vannimenus and G. Toulouse, J. Phys. C 10, L537 (1977).

${ }^{8}$ M. Kac, in Statistical Physics, Phase Transitions and Superfluidity, edited by M. Chretien, E. P. Gross, and S. Deser (Gordon and Breach, New York, 1966), Vol. 1, p. 241.

${ }^{9} \mathrm{~B}$. Derrida, to be published.

${ }^{10}$ R. G. Palmer and C. M. Pond, J. Phys. F $\underline{9}, 1451$ (1979).

${ }^{11}$ G. Parisi, Laboratori Nazionale di Frascati Report No. LNF 79/46 (to be published). 